\title{
Intergroup Rhabdomyosarcoma Group III
}

National Cancer Institute

\section{Source}

National Cancer Institute. Intergroup Rhabdomyosarcoma Group III. NCI Thesaurus.

Code C148019.

Gross residual disease. 